\title{
Hirschsprung associated GDNF mutations do not prevent RET activation
}

\author{
Silvia Borghini ${ }^{1}$, Renata Bocciardi ${ }^{1}$, Giulia Bonardi ${ }^{1}$, Ivana Matera ${ }^{1}$, Giuseppe Santamaria ${ }^{1}$, \\ Roberto Ravazzolo $^{1,2}$ and Isabella Ceccherini ${ }^{\star, 1}$ \\ ${ }^{1}$ Laboratorio di Genetica Molecolare, Istituto G. Gaslini, 16148 Genova, Italy; ${ }^{2}$ Department of Oncology, Biology \\ and Genetics, University of Genova, Italy
}

Hirschsprung disease (HSCR) is a complex disorder characterised by aganglia of distal gastrointestinal tracts. The highest proportion of both familial and sporadic cases is due to mutations of the RET proto-oncogene. Five germline mutations in the glial cell-line-derived neurotrophic factor (GDNF) gene, one of the RET ligands, have been detected in HSCR patients. Pedigrees analysis and the observed association between these GDNF alterations and RET variants in the same patients raised the question of whether the GDNF gene plays any causative/predisposing role in HSCR pathogenesis. In the present work, we have studied the ability of GDNF proteins, each bearing one of the reported mutations, to activate RET by performing a functional test in cultured neuroblastoma cells. Consistently with the lack of genotype/phenotype correlation in human subjects, our results indicate absence of detectable alterations of mutant GDNF induced RET activation. European Journal of Human Genetics (2002) 10, 183 -187. DOI: 10.1038/sj/ejhg/5200785

Keywords: glial cell line-derived neurotrophic factor; RET proto-oncogene; Hirschsprung disease; RET activation assay

\section{Introduction}

Hirschsprung disease (HSCR) or congenital megacolon is a disorder characterised by the absence of intrinsic ganglion cells along variable portions of the distal gastrointestinal tract. From a clinical perspective it presents in the first years of life as severe constipation with abdominal distension, failure to thrive and intestinal obstruction. ${ }^{1}$ HSCR is characterised by variable expressivity, incomplete sex-dependent penetrance and a complex pattern of inheritance due to the involvement of several genes in its pathogenesis. ${ }^{2,3}$ Inactivating, loss-of-function mutations of the RET protooncogene, a gene encoding for a receptor tyrosine kinase expressed during development of neural crest derivatives, can be detected in a proportion of both familial and sporadic HSCR patients. ${ }^{4}$ Additional HSCR susceptibility genes are represented by the G-protein coupled Endothelin B receptor

${ }^{*}$ Correspondence: Isabella Ceccherini, Laboratorio di Genetica Molecolare, Istituto G. Gaslini, L.go G. Gaslini, 5, 16148 Genova, Italy; Tel: +39 010 5636400; Fax: +39 010 3779797; E-mail: isa.c@unige.it Received 15 October 2001; revised 8 January 2002; accepted 16 January 2002
$(E D N R B)$, its ligand Endothelin $3(E D N 3)$, the transcriptional regulator $S O X 10$, the endothelin-converting enzyme (ECE1) and the $S M A D$ interacting protein 1 (SIP1)..$^{5-7}$ However, mutations in these latter genes are rare and generally restricted to HSCR associated with specific phenotypes, like deafness and pigmentary defects or mental retardation and microcephaly. ${ }^{7-10}$

The glial cell line-derived neurotrophic factor $(G D N F)$ is one of the RET ligands. ${ }^{11}$ It is a distantly related member of the transforming growth factor $\beta$ superfamily, as indicated by the presence of a cysteine-knot motif in its quaternary structure. The three-dimensional structure of the GDNF monomere is characterised by two long fingers of antiparallel $\beta$-strands and a $\alpha$-helix at the opposite end. ${ }^{12}$ The mature protein is represented by a homodimer of a 134 residues-long glycosylated polypeptide that is released by $\mathrm{N}$ terminal proteolytic cleavage from a 211 amino acids precursor. It acts in a multi-subunit receptor in which a third component, GDNF receptor $\alpha 1$ (GFRA1), is also required. ${ }^{13,14}$

While no mutation of the GFRA1 gene has been found by extensive screening, ${ }^{15,16}$ five mutations affecting the GDNF coding sequence have been detected to date in a total of 510 
HSCR patients, all screened also for RET mutations. ${ }^{17-24} \mathrm{~A}$ subset of these patients had also been analysed at other HSCR susceptibility loci. ${ }^{21-24}$ Among the GDNF variants so far identified only a missense nucleotide substitution determining the T154S change has been reported as a de novo mutation in a sporadic case of HSCR. ${ }^{17}$ Another missense mutation, leading to R93W, has been found in two HSCR patients in association with a single base pair deletion in the cytoplasmic domain of $R E T^{18}$ and a heterozygous synonymous variant of the $R E T$ gene interfering with mRNA splicing, ${ }^{19}$ respectively. The same substitution has been detected also in a sporadic case of pheochromocytoma ${ }^{25}$ and in a patient with Ondine's curse. $^{26}$ The remaining three GDNF mutations are: a P21S change, found in association with a specific maternal/ paternal combination of haplotypes at the RET locus; ${ }^{19}$ a D150N missense mutation, found in two HSCR patients, one of whom was also affected by Down syndrome, ${ }^{19}$ and in an unaffected control individual; ${ }^{27}$ a I211M change not segregating with the disease phenotype, being inherited by one of two equally affected siblings from the healthy mother. ${ }^{28}$ More recently, a novel $\mathrm{T} \rightarrow \mathrm{C}$ heterozygous mutation has been detected 25 bases upstream from the GDNF coding region in a patient also carrying a missense mutation in exon 12 of the RET gene (T706A). ${ }^{21}$

Finally, a missense mutation in the neurturin gene, encoding another RET ligand, has been reported as not sufficient to cause HSCR disease since a RET missense mutation also segregates in the same family. ${ }^{29}$

The absence of a clear genotype-phenotype correlation of GDNF mutations in HSCR patients and the observed association of GDNF alterations with variants of the RET proto-oncogene suggested a very marginal role of GDNF in the pathogenesis of HSCR. On the other hand, Gdnf -/mice exhibited phenotypes similar to Ret $-/-$ mice, displaying intestinal aganglionosis and renal agenesis. ${ }^{30-}$ 32 Functional studies are therefore necessary to assess the possible causative/predisposing nature of each GDNF variant identified so far in HSCR development. To this end, we have assayed the ability of five different mutated GDNF proteins to activate RET in cultured neuroblastoma cells.

\section{Materials and methods}

\section{Site directed mutagenesis}

GDNF mutant cDNAs were obtained using a commercial kit in accordance with the manufacturer's instructions (Altered sites ${ }^{\circledR}$ in vitro mutagenesis system, Promega). In brief, a cDNA containing the full-length sequence of human GDNF was subcloned into the pAlter vector. Single-stranded cDNA from this construct was used as a template for oligonucleotidebased site-directed mutagenesis, obtaining the following variants: $61 \mathrm{C} \rightarrow \mathrm{T} \quad(\mathrm{P} 21 \mathrm{~S}), \quad 277 \mathrm{C} \rightarrow \mathrm{T} \quad(\mathrm{R} 93 \mathrm{~W}), \quad 448 \mathrm{G} \rightarrow \mathrm{A}$ $(\mathrm{D} 150 \mathrm{~N}), 460 \mathrm{~A} \rightarrow \mathrm{T}(\mathrm{T} 154 \mathrm{~S}), 630 \mathrm{C} \rightarrow \mathrm{G}$ (I211M). All mutations were confirmed by direct sequencing.
Proteins production and quantitative assay

Both the wild-type (wt) and the five mutant GDNF cDNAs were subcloned into the pcDNA3.1 eukaryotic expression vector (Invitrogen). COS7 cells were transiently transfected by using DEAE-dextran-chloroquine method and the six GDNF proteins thus produced were recovered from each conditioned medium. In particular, on the day after transfection, complete medium was changed to serum-free Dulbecco's modified Eagle's medium supplemented with insulin, transferrin and selenium (Gibco BRL). Three days later, conditioned medium was harvested and concentrated 80 - to 150 -fold by ultrafiltration using Centriprep 20 or 80 plus filters (Amicon).

GDNF content in concentrated COS7-conditioned medium was determined using a specific ELISA test following the manufacturer's instructions (GDNF $\mathrm{E}_{\max }{ }^{\text {(iil }}$ ImmunoAssay System, Promega). Conditioned media collected and concentrated from cultured cells were assayed in triplicate and GDNF standards, ranging from 1000 to $0 \mathrm{pg} / \mathrm{ml}$, were prepared using serial dilutions of recombinant human GDNF. Cell derived GDNF levels were determined by interpolation from standard curves assayed on individual plates.

\section{RET phosphorylation assay}

GDNF functional test was performed in SK-N-MC cells, a human neuroblastoma cell line stably transfected with RET cDNA and characterised by endogenous expression of GFRA1. All mutant GDNF proteins were assayed directly from concentrated conditioned medium as already described. ${ }^{33}$ In particular, cells were serum starved for $24 \mathrm{~h}$ prior to use and then treated with various concentrations of wt and mutated GDNF, namely 10, 30 and $100 \mathrm{ng} / \mathrm{ml}$. Recombinant GDNF (R\&D systems) and concentrated medium from mock-transfected cells were always used as positive and negative controls respectively. Treated cells were lysed and immunoprecipitated with rabbit anti-RET polyclonal antibody (Santa Cruz). Immunocomplexes were collected with protein A-sepharose (Amersham), washed, fractionated by $7.5 \%$ SDS-PAGE and transferred to PVDF membrane (Millipore). The extent of RET tyrosine phosphorylation was determined by probing the membrane with a biotin-conjugated antiphosphotyrosine monoclonal antibody (Upstate biotechnology). Detection was achieved using a streptavidin-biotinylated horseradish peroxidase complex (Amersham) and chemiluminescence reagent ECL (Amersham). Stripped blots were reprobed with rabbit anti-RET polyclonal antibody (Santa Cruz).

Autophosphorylation levels and amounts of RET receptor were quantified by image analysis using the ImageQuant software. Values of RET activation, normalised for the corresponding amounts of p170 mature form of RET, were obtained from three tests, performed by treating cells with $30 \mathrm{ng} / \mathrm{ml}$ GDNF, and submitted to statistical analysis. In particular, differences between wt and each mutant GDNF were analysed by the Student's $t$-test. 


\section{Results}

Five different mutant GDNF constructs, obtained by sitedirected mutagenesis from a wild-type (wt) cDNA, have been used to transiently transfect COS7 cells. Both wt and mutant GDNF proteins, secreted in the corresponding conditioned medium, were quantified by ELISA test using purified recombinant GDNF as a standard. The five mutant GDNF proteins under analysis displayed the expected molecular weight on Western blot analysis and could be recovered from COS7 conditioned media at levels comparable with the wt (data not shown).

The ability to activate the RET receptor was assayed for all mutants and wt by assessing the level of the receptor autophosphorylation on tyrosine residues, after using concentrated conditioned medium directly on RET espressing SK-N-MC neuroblastoma cells (see Figure 1 for a representative experiment). Recombinant GDNF was always used in the same assay as a positive control. Concentrated medium from mock-transfected cells had no effect on RET kinase. The test was performed at three different GDNF concentrations: $100 \mathrm{ng} / \mathrm{ml}$ and $30 \mathrm{ng} / \mathrm{ml}$ induced similar levels of receptor activation, while the third one, $10 \mathrm{ng} / \mathrm{ml}$, determined lower autophosphorylation level, presumably reflecting a condition of non complete saturation ${ }^{34}$ (data not shown). The test was repeated three times with the $30 \mathrm{ng} / \mathrm{ml}$ concentration to verify its reproducibility. All the five GDNF mutants under analysis showed a degree of RET autophosphorylation roughly comparable with that induced by the wt GDNF protein. To assess possible slight differences between mutants and $\mathrm{wt}$, signals obtained from the three tests performed at $30 \mathrm{ng} / \mathrm{ml}$ GDNF concentration were quantified by analysing the films with the ImageQuant software. For each experiment, band intensities corresponding to the tyrosine phosphorylated RET were corrected according to the level of the mature p170 RET protein present in each lane of the same blot. To compare each mutant to wt, the normalised values of RET autophosphorylation, shown in Figure 2, were subjected to a $t$-test, thus confirming that none of the tested mutations induced a statistically significant modification of the GDNF ability to activate RET $(P>0.05)$.

\section{Discussion}

Genetic mapping in families and mutation screening of candidate genes have clearly shown the involvement of multiple genes in the pathogenesis of HSCR disease. ${ }^{2,3}$ A wide range of mutations of the $R E T$ proto-oncogene accounts for the highest proportion of both familial and sporadic cases. ${ }^{4}$ Other genes, some to be identified yet, are involved to a lesser extent in determining a HSCR predisposing genetic background. ${ }^{3,5-7}$ Both the identification of GDNF mutations in HSCR patients and the generation of Gdnf - /- mice, characterised by a phenotype similar to Ret -/ - mice, including kidney agenesis, lack of enteric ganglia and pyloric stenosis, ${ }^{30-32}$ suggested a potential involvement of the

\section{IP: anti-RET}

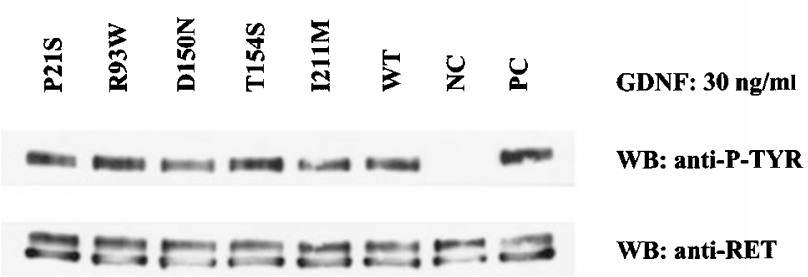

Figure 1 Induction of RET auto-phosphorylation by treatment of SK-N-MC cells with $30 \mathrm{ng} / \mathrm{ml}$ of mutant GDNF proteins. After treatment, RET was immunoprecipitated and blots were probed with anti-phosphotyrosine antibodies (upper) and re-probed with anti-RET antibodies (lower). In the RET blot the two immunobands detected correspond to the mature p170 RET and to the incompletely glysosylated p150 RET precursor, respectively. $\mathrm{NC}=$ negative control (concentrated supernatant from mock-transfected cells). PC=positive control (R\&D systems GDNF).

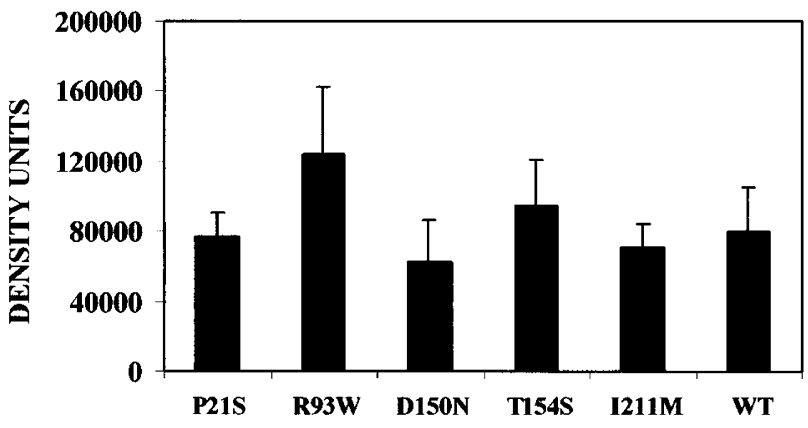

Figure 2 Extent of RET phosphorylation induced by treatments with different mutant GDNF proteins. Quantification is obtained in density units by using the ImageQuant software. Each phosphorylation value is normalised with respect to the corresponding RET amount. Values represent means of three independent experiments carried out by using $30 \mathrm{ng} / \mathrm{ml}$ of the corresponding GDNF protein for cell treatment. Error bars represent standard deviation of the mean.

GDNF gene in HSCR pathogenesis. However, following the observation that GDNF mutations detected so far in HSCR patients are frequently associated with RET mutations and are not segregating with the disease phenotype within families, it has been proposed that they are not sufficient to cause impairment of the normal intestinal innervation. ${ }^{18,19}$ Nevertheless, these GDNF mutations might be relevant, although in combination with other susceptibility factors, in the pathogenesis of HSCR for several reasons: (i) with the exception of the D150N substitution, the other mutations are absent in normal controls; (ii) four mutations affect conserved residues (P21, R93, T154 and I211), and also the fifth one, at codon 150 , changes a residue which, although not conserved, still shows similar chemical features in human (aspartic acid) and in rat (glutamic acid); finally, 
(iii) with the exception of the T154S substitution, the other four mutations lead to variations of chemical and physical features of the corresponding residues.

None of the studies performed so far on the relationship between structure and function of GDNF has reported evidences in favour of a role of the residues involved in HSCR associated GDNF mutations to guarantee RET activation or cell surviving. In the attempt to identify the amino acids directly involved in GFR $\alpha 1$ binding, Eketjall et al $\mathrm{l}^{33}$ mutated, among many others, the residue in position 150 showing a poor effect of this change on GFR $\alpha 1$ binding and no consequences on RET activation. Moreover, a deletion of the GDNF N-terminal end, including the R93 residue, reduced binding to GFR $\alpha 1$ by $\sim 20$-fold, maintaining at the same time the ability to activate RET. ${ }^{33}$ More recently, Baloh et $a l^{35}$ have identified the GDNF regions critical for activating the GFR $\alpha 1 /$ RET and GFR $\alpha 2 /$ RET receptor complexes. According to this study, none of the amino acids involved in HSCR associated GDNF mutations is included in the GFR $\alpha 1 /$ RET critical region, whereas D150 and T154 are included in the GFR $\alpha 2 /$ RET critical region. However, it is difficult to attribute a role to the D150N and T154S changes in HSCR development since, although an interaction between GDNF and GFR $\alpha 2$ is detectable in vitro, this co-receptor is considered a specific partner of neurturin in vivo. ${ }^{36}$ Finally, Chen et al ${ }^{37}$ have demonstrated that deletions including the T154 and I211 residues abolished the GDNF ability to maintain motor neurons from the spinal cord. However, since such deletions also lack the complete $\alpha$-helix and the finger domain fundamental to activate the GFR $\alpha 1 /$ RET receptor complex, their effect on GDNF activity is likely to be dependent on deprivation of whole structural regions rather than on the loss of single residues.

Based on the above considerations, in the present work we have investigated the functional effect of GDNF mutations observed in HSCR patients, as a means to establish their possible contribution to the HSCR phenotype. As mentioned above, Eketjall et al ${ }^{33}$ showed that specific GDNF mutations alter the GFR $\alpha 1$ binding but retain the ability to activate RET at normal levels, concluding that probably there are two binding sites for GDNF, one formed by GFR $\alpha 1$ and one formed by GFR $\alpha 1 /$ RET complex. They hypothesised different signalling capabilities for these two sites ${ }^{33}$ and, in fact, RETindependent GDNF signalling has been shown in neuronal cells. ${ }^{38}$ However, since no mutation of the GFRA1 gene has been found so far in HSCR patients, in spite of extensive screenings, ${ }^{15,16}$ it is questionable whether this alternative pathway has any role in the development of HSCR disease. For this reason, we decided to functionally test HSCR associated GDNF mutations by analysing the ability of GDNF mutants to induce RET activation in intact cells. This is the first step of a signal cascade finalised to transcriptional activation in the nucleus. Normal levels of GDNF induced RET autophosphorylation are assumed as the evidence of normal downstream RET-dependent pathway.
After producing five GDNF mutant proteins in COS7 cells and testing their effect on RET expressing SK-N-MC neuroblastoma cells, we have observed a degree of receptor autophosphorylation comparable to that induced by the wt GDNF protein at any tested concentration. A statistical analysis has confirmed that the variability observed in the extent of mutants and wt GDNF induced RET autophosphorylation, both within the same blots and among different blots, is not due to a significant difference in their ability to activate RET (Figure 2).

Our attempt of quantifying the GDNF induced RET activation might have missed faint differences between wt and some of the mutant GDNF proteins. We believe that such a putative undetected effect of mutant proteins, if present, could have been so low that its contribution to the resulting intestinal phenotype would be similarly negligible.

While in our functional assay mutant GDNF proteins, produced in the absence of the wt construct, could be only tested as mutant homo-dimers, in HSCR patients GDNF mutations have been detected at the heterozygous status, thus raising the possibility of an in vivo dominant negative effect of mutant/wt hetero-dimers. However, having excluded any role of the mutant homo-dimers, any additional effect of mutant GDNFs can be considered as unlikely.

Finally, the analysed GDNF mutations may have a functional relevance, which we could not rule out, in specific $R E T$ contexts, such as common RET haplotypes or SNP alleles. On the other hand, the lack of association with RET mutations affecting the extracellular, GDNF interacting domain seems to exclude a direct, synergical effect of reported GDNF mutations and RET variants on the phenotype.

In conclusion, we have demonstrated that none of the GDNF mutations observed in HSCR patients significantly prevents RET activation and, presumably, the following signal transduction in cultured neuroblastoma cells. This observation is consistent with the lack of genotype/ phenotype correlation in human subjects.

\section{Acknowledgments}

We thank Dr Meitinger and Lena Grimm for their kind gift of clones containing the GDNF cDNA and Gianluca Caridi for help in image analysis. The financial support of Telethon Italy (grant no.E791) is gratefully acknowledged. This work is also supported by the European Community (contract no. QLG1-CT-2001-01646).

\section{References}

1 Puri P: Hirschsprung's disease: clinical generalities; in Holschneider AM, Puri P (eds): Hirschsprung's disease and allied disorders. Singapore: Harwood Academic Publishers, 2000, pp 129-136.

2 Badner JA, Sieber WK, Garver KL, Chakravarti A: A genetic study of Hirschsprung disease. Am J Hum Genet 1990; 46: 568-580.

3 Bolk S, Pelet A, Hofstra RM et al: A human model for multigenic inheritance: phenotypic expression in Hirschsprung disease requires both the RET gene and a new $9 \mathrm{q} 31$ locus. Proc Natl Acad Sci USA 2000; 97: 268-273. 
4 Seri M, Yin L, Barone V et al: Frequency of RET mutations in long- and short-segment Hirschsprung disease. Hum Mutat 1997; 9: 243-249.

5 Parisi MA, Kapur RP: Genetics of Hirschsprung disease. Curr Opin Pediatr 2000; 12: 610-617.

6 Ceccherini I, Attié T, Martucciello G, Nihoul-Fékété C, Lyonnet S, Romeo G: The molecular genetics of Hirschsprung's disease; in Holschneider AM, Puri P (eds): Hirschsprung's disease and allied disorders, Singapore: Harwood Academic Publishers, 2000, pp 69-88.

7 Wakamatsu N, Yamada Y, Yamada K et al: Mutations in SIP1, encoding Smad interacting protein-1, cause a form of Hirschsprung disease. Nat Genet 2001; 27: 369-370.

8 Pingault V, Bondurand N, Kuhlbrodt Ket al: SOX10 mutations in patients with Waardenburg-Hirschsprung disease. Nat Genet 1998; 18: $171-173$

9 Edery P, Attie T, Amiel J et al: Mutation of the endothelin-3 gene in the Waardenburg-Hirschsprung disease (Shah-Waardenburg syndrome). Nat Genet 1996; 12: $442-444$

10 Hofstra RM, Osinga J, Tan-Sindhunata G et al: A homozygous mutation in the endothelin-3 gene associated with a combined Waardenburg type 2 and Hirschsprung phenotype (Shah-Waardenburg syndrome). Nat Genet 1996; 12: $445-447$.

11 Trupp M, Arenas E, Fainzilber $\mathrm{M}$ et al: Functional receptor for GDNF encoded by the c-RET proto-oncogene. Nature 1996; 381: $785-789$.

12 Eigenbrot C, Gerber N. X-ray structure of glial cell-derived neurotrophic factor at $1.9 \AA$ resolution and implications for receptor binding. Nat Struct Biol 1997; 4: 435-438.

13 Treanor JJ, Goodman L, de Sauvage F et al: Characterization of a multicomponent receptor for GDNF. Nature 1996; 382: 80-83.

14 Jing S, Wen D, Yu Y et al: GDNF-induced activation of the RET protein tyrosine kinase is mediated by GDNFR-alpha, a novel receptor for GDNF. Cell 1996; 85: 1113-1124.

15 Angrist M, Jing S, Bolk S et al: Human GFRA1: cloning, mapping, genomic structure, and evaluation as a candidate gene fo Hirschsprung disease susceptibility. Genomics 1998; 48: $354-$ 362

16 Myers SM, Salomon R, Goessling A et al: Investigation of germline GFR alpha-1 mutations in Hirschsprung disease. $J$ Med Genet 1999; 36: 217-220.

17 Ivanchuck SM, Myers SM, Eng C, Mulligan LM. De novo mutation of GDNF ligand for the RET/GDNFR-alpha receptor complex in Hirschsprung disease. Hum Mol Genet 1996; 5: 2023-2026.

18 Angrist M, Bolk S, Halushka M, Lapchak PA, Chakravarti A. Germline mutations in glial cell line-derived neurotrophic factor GDNF and RET in a Hirschsprung disease patient. Nat Genet 1996; 14: $341-344$.

19 Salomon R, Attie T, Pelet A et al: Germline mutations of the RET ligand GDNF are not sufficient to cause Hirschsprung disease. Nat Genet 1996; 14: $345-347$

20 Hofstra RM, Wu Y, Stulp RP et al: RET and GDNF gene scanning in Hirschsprung patients using two dual denaturing gel systems. Hum Mutat 2000; 15: $418-429$.

21 Sakai T, Nirasawa Y, Itoh Y, Wakizaka A: Japanese patients with sporadic Hirschsprung: mutation analysis of the receptor tyrosine kinase proto-oncogene, endothelin-B receptor, endothelin-3, glial cell line-derived neurotrophic factor and neurturin genes: a comparison with similar studies. Eur J Pediatr 2000; 159: $160-167$
22 Gath R, Goessling A, Keller KM et al: Analysis of the RET, GDNF, EDN3, and EDNRB genes in patients with intestinal neuronal dysplasia and Hirschsprung disease. Gut 2001; 48: 671-675.

23 Munnes M, Fanaei S, Schmitz B, Muiznieks I, Holschneider AM, Doerfler W: Familial form of Hirschsprung disease: nucleotide sequence studies reveal point mutations in the RET protooncogene in two of six families but not in other candidate genes. Am J Med Genet 2000; 94: 19-27.

24 Borrego S, Eng C, Sanchez B, Saez ME, Navarro E, Antinolo G: Molecular analysis of the RET and GDNF genes in a family with multiple endocrine neoplasia type2A and Hirschsprung disease. J Clin Endocrinol Metab 1998; 83: 3361 - 3364.

25 Woodward ER, Eng C, McMahon R et al: Genetic predisposition to phaeochromocytoma: analysis of candidate genes GDNF, RET and VHL. Hum Mol Genet 1997; 6: 1051-1056.

26 Amiel J, Salomon R, Attie T et al: Mutations of the RET-GDNF signaling pathway in Ondine's curse. Am J Hum Genet 1998; 62: $715-717$.

27 Hofstra RMW, Osinga J, Buys C: Mutations in Hirschsprung Disease: when does a mutation contribute to the phenotype. Eur J Hum Genet 1997; 5: 180 - 185.

28 Martucciello G, Thompson H, Mazzola C et al: GDNF deficit in Hirschsprung's disease. J Pediatr Surg 1998; 33: 99-102.

29 Doray B, Salomon R, Amiel J et al: Mutation of the RET ligand, neurturin, supports multigenic inheritance in Hirschsprung disease. Hum Mol Genet 1998; 7: 1449-1452.

30 Sanchez MP, Silos-Santiago I, Frisen J, He B, Lira SA, Barbacid M: Renal agenesis and the absence of enteric neurons in mice lacking GDNF. Nature 1996; 382: 70-73.

31 Pichel JG, Shen L, Sheng HZ et al: Defects in enteric innervation and kidney development in mice lacking GDNF. Nature 1996; 382: $73-76$

32 Moore MW, Klein RD, Farinas I et al: Renal and neuronal abnormalities in mice lacking GDNF. Nature 1996; 382: 76-79.

33 Eketjall S, Fainzilber M, Murray-Rust J, Ibanez CF: Distinct structural elements in GDNF mediate binding to GFRa1 and activation of the GFRa1/c-RET receptor complex. EMBO J 1999; 18: $5901-5910$.

34 Worby CA, Vega QC, Zhao Y, Chao HH, Seasholtz AF, Dixon JE: Glial cell line-derived neurotrophic factor signals through the RET receptor and activates mitogen-activated protein kinase. $J$ Biol Chem 1996; 271: 23619-23622.

35 Baloh RH, Malù GT, Eugene MJ, Milbrant J: Functional mapping of receptor specificity domains of glial cell line-derived neurotrophic factor (GDNF) family ligands and production of GFR $\alpha 1$ RET-specific agonists. J Biol Chem 2000; 275: 3412 - 3420.

36 Rossi J, Luukko K, Poteryaev D et al: Retarded growth and deficits in the enteric and parasympathetic nervous system in mice lacking GFRalpha2, a functional neurturin receptor. Neuron 1999; 22: 243-252.

37 Chen ZY, He ZY, He C, Lu CL, Wu XF: Human glial cell-linederived neurotrophic factor: a structure-function analysis. Biochem Biophys Res Commun 2000; 268: 692-696.

38 Trupp M, Scott R, Whittemore SR, Ibanez CF: Ret-dependent and -independent mechanisms of glial cell line-derived neurotrophic factor signaling in neuronal cells. J Biol Chem 1999, 274: 20885 - 20894. 